\title{
Spectral Efficiency Of QPSK In A Coherent WDM System With Component Crosstalk
}

Pinakpani Mukherjee ( $\nabla$ ppmukherjee@yahoo.co.in )

AOT: Academy of Technology https://orcid.org/0000-0001-7157-8545

\section{Santu Sarkar}

University of Calcutta

Nikhil R. Das

University of Calcutta

\section{Research Article}

Keywords: Wavelength division multiplexing, spectral efficiency, QPSK, component crosstalk, interference penalty, optical network, SNR, BER, coherent detection.

Posted Date: March 7th, 2022

DOI: https://doi.org/10.21203/rs.3.rs-1403576/v1

License: @ (i) This work is licensed under a Creative Commons Attribution 4.0 International License.

Read Full License 


\title{
Spectral efficiency of QPSK in a coherent WDM system with component crosstalk
}

\author{
Pinakpani Mukherjee ${ }^{1}$, Santu Sarkar ${ }^{2}$ and Nikhil R. Das ${ }^{3}$ \\ ${ }^{1}$ Dept. of Electronics \& Communication Engineering, Academy of Technology, Adisaptagram, Hooghly, India \\ ${ }^{2,3}$ Institute of Radio Physics and Electronics, University of Calcutta, 92 A.P.C. Road, Kolkata, India \\ 1 ppmukherjee@yahoo.co.in, ${ }^{2}$ santu@ieee.org, ${ }^{3}$ nrd@ieee.org
}

Abstract: - This paper presents an analytical method to investigate the impact of component crosstalk on the spectral efficiency (SE) of generalized quadrature phase shift keying (QPSK) modulation format by computing its bit error rate (BER) over additive white Gaussian noise (AWGN) channel. For this purpose we consider a wavelength division multiplexed (WDM) receiver system and the desired signal is coherently detected in presence of any arbitrary number of independent and identical (i.i.d.) crosstalk sources. The analysis exploits characteristic function method and examines the bandwidth expenses of the system while subjected to crosstalk sources. The theory is verified for single interferer case with the previous work reported in literature. The approach is useful for accurate and time-saving BER (thus SE) assessment and monitoring in the network/system, which can improve the reach and efficiency of the WDM system. It is observed from the result that crosstalk level and number of active interferers perform a vital role to determine the spectral efficiency of the system. In this context the study of the protection ratio i.e. the minimum crosstalk level required to obtain a fixed BER, is essential for any particular signal to noise ratio (SNR). The power budgeting of the network under component crosstalk is also discussed in terms of spectral efficiency and interference penalty.

Index Terms-Wavelength division multiplexing, spectral efficiency, QPSK, component crosstalk, interference penalty, optical network, SNR, BER, coherent detection.

\section{INTRODUCTION}

oherent optical detection along with advanced modulation format is a promising candidate to achieve long-haul and high spectral efficiency fiber transmission systems [1]-[2]. With the recent trend for increased spectral efficiency and greener communications, a search for power-efficient modulation formats for coherent transmission is going on and a class of fourdimensional (4-D) signal constellations is proposed as an outcome. In this context several forms of QPSK (quadrature phase shift keying) are investigated which boasts an advantage to 
achieve such goals [3]-[4]. Recently in case of metro or access network also, coherent detection is considered for possible application [1].

Multi wavelength optical networks where the wavelength routers are used for channel routing and add-drop are the basis of future information infrastructure. A fundamental difficulty of wavelength routers are crosstalk/ interference which originates from neighbouring inputs. In the case, where the crosstalk signals have similar or identical frequency/wavelength to the signal channel, the degradation is more severe as it is not possible to eliminate through filtering. This undesired phenomenon is termed as homodyne or component crosstalk in optical domain and belongs to linear crosstalk category [5]. In wireless communication system, this difficulty is termed as cochannel interference which is mathematically similar to homodyne crosstalk [6]. The severity of degradation due to component crosstalk in an optical network is normally assessed in terms of few parameters, namely bit error rate (BER), spectral efficiency (SE), interference/power penalty (IP) etc.

Component crosstalk for optical network was first reported in [7]. After that analysis was shifted towards various digital modulation schemes (like on off Keying (OOK), phase shift keying (PSK) etc.) in conjunction with different detection technique like directdetection, coherent, matched-filter etc. [5]-[19]. Initially the studies of component crosstalk were largely based on Gaussian approximation even for single interferer, after that the utility of non Gaussian models were also explained [6]. It is well established fact that Gaussian model overestimates in presence of single or small number of interferers, but in practice the network under component crosstalk may be dominated by single or few number of interferers. In recent time $M$-PSK i.e. $M$-ary phase shift keying (e.g. BPSK (binary PSK), QPSK etc.) are being examined for superior crosstalk tolerance and high spectral efficiency [8]-[10]. The instances of these modulation schemes in presence of either component crosstalk or cochannel interference are available in both optical and wireless communication domain literatures [11]-[26]. Here we consider generalized QPSK as topic of interest of this paper, and the related works are included as following. In wireless cases normally polarization is not considered and mostly matched filter reception is studied with different fading environment [20]-[24]. In such a work [20], the performance of BPSK and QPSK with coherent detection and matched filtering are investigated in the presence of both time and phase asynchronous cochannel interfering signals. But the analysis does not deliver any closed form expression for BER in presence of multiple interferers. A new analytical method for computing the average error probabilities in binary and generalized quadriphase signals in presence of Rayleigh-faded cochannel interference is discussed in [23]. However the work is 
meant for diversity receivers. Bandwidth efficient QPSK in cochannel interference and fading is analyzed by N.C. Beaulieu et.al. [24] where an approximate expression for the BER is derived using Fourier series approach. In case of optical domain, effects of homodyne crosstalk on dual polarization QPSK signal is reported by K. P. Ho considering simple matched filter model [26]. In a recent work [27], an analytical approach is presented to find the effect of cross-polarization induced crosstalk on the BER performance of a polarization division multiplex (PDM) QPSK coherent homodyne optical transmission system with polarization diversity receiver.

In [8]-[10], [28]-[29], the information-theoretic limits to SE are discussed considering various detection techniques, modulation techniques and propagation regimes. In such instances, the effect of interference/crosstalk is not considered, and the deviation of SE in presence of crosstalk sources is not studied. Accurate and time-saving BER and SE monitoring is an indispensable function to support software-defined optical network which is the future of the next generation dynamic and flexible networking scenarios [1]. The contributions of this paper toward this goal are as follows. We are presenting a new mathematical formulation to estimate BER for QPSK signal transmission, while detected in a coherent receiver for wavelength division multiplexed (WDM) system. A non Gaussian model exploiting characteristic function method is followed to formulate BER and the approach results in an exact explicit expression for BER. The resultant closed form expression is readily computed in terms of the coefficients of Hermite polynomial. It accounts for the contribution of any arbitrary number of component crosstalk sources and applicable over additive white Gaussian noise (AWGN) channels. From the knowledge of the estimated BER in presence of specific crosstalk sources, the deviation in SE is computed to understand the additional bandwidth and power expenses. This analysis will be helpful for assessing BER, along with corresponding penalty induced to the practical networks under component crosstalk and can be treated as a theoretical benchmark of assessment while testing.

The rest of the paper is organized as follows. The mathematical formulation of BER is described in section II. Results obtained from the analysis are discussed in section III. Finally, conclusion is given. 


\section{MATHEMATICAL FORMULATION}

Considering an $M$-ary PSK signal having a constant amplitude of $A$ and $M$ evenly spaced phases of $\theta_{s}=\frac{\pi(2 s-1)}{M}$ where $s=1,2,3 \ldots . . M$; the $M$ different signal waveforms can be written as $[6] \ldots$.

$$
\begin{aligned}
& \xi_{s}(t)=\Re\left\{A \exp \left(\frac{j \pi(2 s-1)}{M}\right) \exp \left(j \omega_{c} t\right)\right\} \\
& =A \cos \left[\frac{\pi(2 s-1)}{M}\right] \cos \omega_{c} t-A \sin \left[\frac{\pi(2 s-1)}{M}\right] \sin \omega_{c} t
\end{aligned}
$$

Here $\omega_{c}$ is carrier frequency. The Euclidean distance [6] between the $s$ and $t$-th symbols is equal to $\sqrt{2} A|\sin ((s-t) \pi / M)|$, where the minimum distance is $\sqrt{2} A|\sin (\pi / M)|$.

For a QPSK signal the value of $M$ is 4 and corresponding signal is given by..

$$
\xi_{s}(t)=A \cos \left[\frac{\pi(2 s-1)}{4}\right] \cos \omega_{c} t-A \sin \left[\frac{\pi(2 s-1)}{4}\right] \sin \omega_{c} t
$$

where $s=1,2,3$ and 4. QPSK modulation format have four symbols where each symbols consist of two bits and there is four unique phases (e.g. $\theta_{S}=\pi / 4,3 \pi / 4,5 \pi / 4$ and $7 \pi / 4$ ). Amplitude $(A)$ can be expressed in terms of energy $(E)$ and time duration $(T)$ of each symbol, as following.

$$
\begin{aligned}
& E=2 E_{b} \& T=2 T_{b} \\
& A=\sqrt{2 E / T}=\sqrt{2 E_{b} / T_{b}}
\end{aligned}
$$

where $E_{b}$ and $T_{b}$ are energy and time duration of each bit respectively. Thus for the values of $s=1,2,3$ and 4 , in general (2) can be written as....

$$
\begin{aligned}
& \xi_{s}(t)= \pm \frac{A}{\sqrt{2}} \cos \omega_{c} t \pm \frac{A}{\sqrt{2}} \sin \omega_{c} t \\
& =\xi_{s}^{i} \sin \omega_{c} t+\xi_{s}^{q} \cos \omega_{c} t
\end{aligned}
$$

In (4), $\xi_{s}^{i}$ and $\xi_{s}^{q}$ are the baseband signals on the in-phase and quadrature paths [25].

Let us consider a QPSK signal, sent from one node (source) to another node (destination) in an optical network, subjected to component crosstalk (similar to cochannel interference). Let $\xi_{s}(t)$ be the desired QPSK signal for transmission as expressed by (4).The crosstalk signals from the neighboring nodes at same nominal frequency $\left(\omega_{c}\right)$ are also assumed QPSK in nature and are given by

$$
\xi_{i}(t)=\sqrt{\varepsilon_{i}}\left[\xi_{i}^{i} \sin \left(\omega_{c} t+\phi_{i}\right)+\xi_{i}^{q} \cos \left(\omega_{c} t+\phi_{i}\right)\right]
$$


In (5), $\xi_{i}^{i}$ and $\xi_{i}^{q}$ represent the baseband in-phase and quadrature components of the $i$-th interfering signal respectively. Here $\varepsilon_{i}$ is the crosstalk level, $\phi_{i}(t)$ is the random phase and $i=1,2 \ldots . N$. In the analysis we assume that the crosstalk is perfectly coherent with the signal of interest. Assuming that $N$ crosstalk sources are present, the total received signal $r(t)$ can be written as....

$r(t)=\xi_{s}(t)+\sum_{i=1}^{N} \xi_{i}(t)+n_{g}(t)$

where $\xi_{s}(t)$ and $\xi_{i}(t)$ represent the contributions from the desired signal and the $i$-th interfering signal, respectively and $n_{g}(t)$ is a zero mean AWGN with two sided power spectral density of $N_{0} / 2 \mathrm{~W} / \mathrm{Hz}$. For optimum coherent detection receiver, the total received signal is split into an in-phase and quadrature component, after that detection is performed. We follow a typical receiver structure reported at [25] for the detection purpose. In the following analysis, only the detection in the in-phase path is presented as the results for the quadrature component is similar by symmetry. The in-phase demodulated signal component is given by [25]

$$
D_{a}(t)=2 r(t) \sin \omega_{c} t
$$

In case of optimum coherent detection, quadrature carriers are locally generated with a phase that approximates the desired received signal. Then the received signal is multiplied by this locally generated quadrature carriers. In this paper for the sake of simplicity perfect carrier phase tracking is assumed for the desired signal. It is also assumed that frequency tracking and symbol synchronization are perfect for the desired signal. Using (6), (7) can be written as...

$$
\begin{aligned}
& D_{a}(t)=\xi_{s}^{i}\left[1-\cos \left(2 \omega_{c} t\right)\right]+\xi_{s}^{q} \sin \left(2 \omega_{c} t\right) \\
& +\sum_{i=1}^{N} \sqrt{\varepsilon_{i}}\left\{\xi_{i}^{i}\left[\cos \phi_{i}-\cos \left(2 \omega_{c} t+\phi_{i}\right)\right]+\xi_{i}^{q}\left[\sin \left(2 \omega_{c} t+\phi_{i}\right)-\sin \left(\phi_{i}\right)\right]\right\} \\
& +2 n_{g}(t) \sin \left(\omega_{c} t\right)
\end{aligned}
$$

Then the signal given by (8), is passed through a square root raised cosine low pass filter with excess bandwidth having a roll of factor $\beta(0 \leq \beta \leq 1)$. The frequency response of this filter is zero outside the frequency interval $\left[-\frac{1+\beta}{2 T}, \frac{1+\beta}{2 T}\right]$. Hence the double frequency signal term on (8) can be eliminated for $f_{c} \gg \frac{1}{2 T}$ and $\beta=0$. The signal at the output of the receiver i.e. $X_{a}(t)$ can be written as ..

$$
X_{a}(t)=\xi_{s}^{i}++\sum_{i=1}^{N} \sqrt{\varepsilon_{i}}\left[\xi_{i}^{i} \cos \phi_{i}-\xi_{i}^{q} \sin \left(\phi_{i}\right)\right]+n_{g}(t)
$$

Here we assumed that the filter have unity gain inside the pass band. Thus, using the noise $n_{g}(t)$ of the receiver itself, the overall signal-crosstalk noise due to $N$ number of interfering 
channels is given by

$n(t)=\sum_{i=1}^{N} \sqrt{\varepsilon_{i}}\left[\xi_{i}^{i} \cos \phi_{i}-\xi_{i}^{q} \sin \left(\phi_{i}\right)\right]+n_{g}(t)$.

$n_{g}(t)$ is the usual Gaussian noise at the receiver with variance and characteristic function of $\sigma_{0}^{2}=N_{0} / 2[12]$ and $\exp \left(-\sigma_{0}^{2} w^{2} / 2\right)$ respectively. Now assuming single interferer case (i.e. $N=1)$ and excluding $n_{g}(t)$, (10) can be written as

$$
\begin{aligned}
& n_{1}(t)=\sqrt{\varepsilon_{1} E_{b} / T_{b}}\left[ \pm \sqrt{1 / 2} \cos \phi_{1} \pm \sqrt{1 / 2} \sin \phi_{1}\right] \\
& =Z_{1} \cos \left(\phi_{1}+\alpha\right)
\end{aligned}
$$

where $Z_{1}=\sqrt{2 \varepsilon_{1} E_{b} / T_{b}}, \alpha \in\left\{ \pm \frac{\pi}{4}, \pm \frac{3 \pi}{4}\right\}$ and $\phi_{1}$ is uniformly distributed in $[0,2 \pi]$. The probability density function (p.d.f.) of $Z \cos [\phi(t)]$ is given by $p(x)=(1 / \pi)\left(Z^{2}-x^{2}\right)^{1 / 2}$ for $Z<x<+Z$ which provides characteristic function of $J_{0}(Z w)$, where $J_{0}($.$) is the Bessel function$ [11].

The analysis used for single interferer in previous paragraph, can be extended to deal with many interferers. In order to calculate the BER, the p.d.f. and characteristic function of $n(t)$ has to be computed. Using (10) and (11) the overall characteristic function due to $N$ number of interferers i.e. $\psi_{n}(w)$, can be written as

$\Psi_{n}(w)=\prod_{i=1}^{N} J_{0}\left(Z_{i} w\right) \exp \left(-\sigma_{0}^{2} w^{2} / 2\right)=\left\{J_{0}\left(Z_{i} w\right)\right\}^{N} \exp \left(-\sigma_{0}^{2} w^{2} / 2\right)$

where $Z_{i}=\sqrt{2 \varepsilon_{i} E_{b} / T_{b}}$. To obtain the expression of characteristic function given by (12), the contribution of all interfering channels are assumed equal and mathematical approach adopted in [11] is followed. The characteristic function due to the presence of arbitrary $N$ number of interferers i.e. $\left\{J_{0}\left(Z_{i} w\right)\right\}^{N}$, can be expanded through the formula based on alternative derivation of the Maclaurin series expansion for the powers of zero order Bessel Function $\left(J_{O}().\right)$ reported in [30]. The formula is valid for any arbitrary number, real or complex .This formulation helps to obtain a new closed form expression of BER for QPSK transmission, which is useful to estimate the impact of finite crosstalk sources. After expansion (12) can be expressed as

$$
\begin{aligned}
& \Psi_{n}(w)=\left[1+\sum_{k=12^{2 k}(k !)^{2}}^{\infty} \frac{(-1)^{k}}{2}(N) \cdot\left(Z_{i} w\right)^{2 k}\right] \exp \left(-\sigma_{0}^{2} w^{2} / 2\right) \\
& =\varphi_{n}^{N}\left(Z_{i} w\right) \exp \left(-\sigma_{0}^{2} w^{2} / 2\right)
\end{aligned}
$$

In (13), $\varphi_{n}^{N}\left(Z_{i} w\right)$ is the characteristic function due to the presence of arbitrary $N$ number of identical interferers and $B_{k}^{0}(N)$ is recursive polynomial as per [30]. The overall p.d.f. can be obtained taking inverse Fourier Transform of (13). 
$p_{n}(r)=\frac{1}{\sqrt{2 \pi \sigma_{0}^{2}}} \exp \left\{(r)^{2} / 2 \sigma_{0}^{2}\right\}+\frac{1}{\sqrt{2 \pi \sigma_{0}^{2}}} \sum_{k=1}^{\infty} \frac{B_{k}^{0}(N) \cdot\left(\frac{Z_{i}^{2}}{2 \sigma_{0}^{2}}\right)^{k}}{2^{k}(k !)^{2}} H e_{2 k}\left(\frac{r}{\sigma_{0}}\right) \exp \left\{(r)^{2} / 2 \sigma_{0}^{2}\right\}$

where $\mathrm{He}_{n}(z)$ is Probabilists' Hermite polynomial [30]. In the derived expression of (14), there are two terms in right hand side. The first term is the conventional p.d.f. expression for BER of QPSK in additive white Gaussian noise (AWGN) with no crosstalk sources. The second term is due to the effect of arbitrary $N$ number of crosstalk sources which consists of a summation term including the polynomial $B_{k}^{0}(N)$ and Hermite polynomial. $H e_{2 k-1}($.$) . The value$ of this second term is dependent on variable $k$, which ranges from one to infinity. It is computationally observed that, there is a specific finite value of $k$, up to which significant contribution of parameter $k$ is observed. Beyond this value of $k$ no significant change in the total summation value is visible. This special finite value of $k\left(k_{\max }\right)$ is dependent on the number of interferers present (i.e. $N$ ). For the computation purpose, $k_{\max }=60$ has been used throughout this paper as discussed in [11]. The expression of (14), is plotted in Fig.1 as a function of random variable $(r)$ which represents the contribution of crosstalk interferers and confirms the non Gaussian nature of the model.

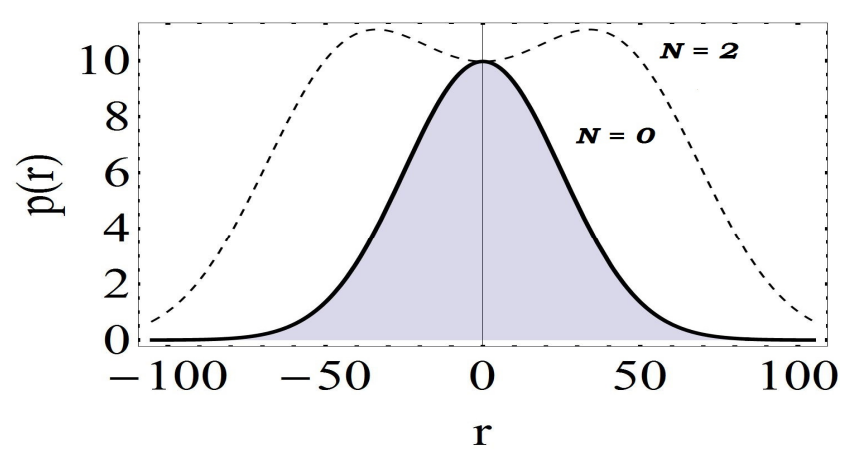

Fig.1:-.Probability density function for $N=2$ interferers (dashed curve) compared with the Gaussian approximation at no crosstalk case (solid filled).

For the derivation of BER, we follow the characteristic function method [20] as it will be more convenient from numerical point of view. Let us assume that the in-phase and quadrature components are mapped together to complex symbols using Gray coding. Following the symmetry of the associated random variables, the bit error probability can be expressed as [21], [31]

$$
P_{e b}=\frac{1}{2 \pi} \int_{-\infty}^{\infty} \psi_{n}(w) \int_{-\infty}^{-A / \sqrt{2}} \exp \{-j w z\} d z d w
$$


Without crosstalk/interference, $\varphi_{n}^{N}\left(Z_{i} w\right)=1$ and consequently from (15) the BER can be written as [21]

$$
\begin{aligned}
& P_{e b}=\frac{1}{2} \operatorname{erfc}\left(\sqrt{E_{b} / N_{0}}\right) \\
& +\frac{1}{\pi} \int_{0}^{\infty}\left[1-\varphi_{n}^{N}\left(Z_{i} w\right)\right] \frac{\sin (w A / \sqrt{2)}}{w} \exp \left(-\sigma_{0}^{2} w^{2} / 2\right) d w
\end{aligned}
$$

In (17), $E_{b} / N_{0}(\rho$ in alternate notation) is signal to noise ratio $(S N R)$ and $\varphi_{n}^{N}\left(Z_{i} w\right)=1+\sum_{k=1}^{\infty} \frac{(-1)^{2}}{2^{2 k}(k !)^{2}} B_{k}^{0}(N) \cdot\left(Z_{i} w\right)^{2 k}$, as shown in (13). After some algebra (16) can be expressed as (17) which is the final closed form expression for BER of interest.

$$
\begin{aligned}
& P_{e b}=\frac{1}{2} \operatorname{erfc}(\sqrt{\rho}) \\
& +\frac{1}{\sqrt{2 \pi}} \sum_{k=1}^{\infty} \frac{B_{k}^{0}(N) \cdot\left(2 \varepsilon_{i} \rho / T_{b}\right)^{k}}{2^{k}(k !)^{2}} H e_{2 k-1}\left(\sqrt{2 \rho / T_{b}}\right) \exp \left(-\rho / T_{b}\right)
\end{aligned}
$$

In (17), $\rho$ is signal to noise ratio $(S N R)$ and $\operatorname{erfc}($.$) is complimentary error function [31].$

The interference penalty is defined as the increase in the SNR required by a system with crosstalk in order to maintain the same BER as a system without crosstalk. From (17), interference penalty $(I P)$ can be calculated using [5]

$$
I P=10 \log _{10}\left(\sqrt{\rho / \rho_{N C}}\right)
$$

where $\rho_{N C}$ and $\rho$ is the SNR of the receiver without and with crosstalk respectively at a specific BER.

The term spectral efficiency $(\eta)$ is used to describe the rate of information/data $(R)$ being transmitted over a given bandwidth $(B)$ in specific communication systems. Spectral efficiency may also be called bandwidth efficiency and usually expressed in bits per second per Hertz $(b / s / H z)$. Channel capacity $(C)$ is governed by maximum data rate and $R \leq C$, so $\eta=C / B$. Assuming a binary symmetric channel (BSC) [33], the capacity with error probability $\left(P_{e}\right)$ is given by [28]

$\eta=1-h\left(P_{e}\right)$ bits

where $h(p)=-p \log _{2}(p)-(1-p) \log _{2}(1-p)$ is the Binary Entropy Function. Using the value of error probability calculated through (17), the spectral efficiency $(\eta)$ can be obtained for any fixed crosstalk level, data rate and SNR in presence of finite component crosstalk sources. Thus the bandwidth efficiency of the system can be examined through (17) and (19). 


\section{RESULT AND DISCUSSION}

With the intention of evaluating the impact of component crosstalk in AWGN, we start by examining the expression of (17), (18) and (19) as obtained in previous section; as a function of different parameters like $S N R$, crosstalk level, thermal noise, number of interfering channels etc. In this regard BER computed using the present model is plotted and compared with the available data from previous literature. In Fig. 2, the values of BER obtained through (17) is compared with the outcome of [26] for worst polarized QPSK signal in presence of single interfering channel and a good agreement has been found.

The variation of BER as a function of SNR is shown in Fig. 3 in presence of various crosstalk sources $(N)$ for fixed crosstalk levels $\left(\varepsilon_{i}\right)$. Absence of crosstalk is indicated by $N=$ 0 . It is clear from the graph that impact of crosstalk is more pronounced in case of medium to high values of SNR.

It is noteworthy to mention that, the presence of few or many crosstalk sources lead to different BER scenario which results in significant variation in the permissible crosstalk protection ratio. Crosstalk protection ratio is the minimum crosstalk level to ensure a fixed BER and is crucial for real time BER monitoring of networks [22]. For the better clarity of this point, crosstalk level required to obtain BER of $10^{-5}$ is plotted in Fig. 4 as a function of $S N R$ with $N=1$ and 5 crosstalk sources. The graph reveals the value of required protection ratio at any specific $S N R$ for any arbitrary crosstalk sources. For example, to obtain BER of $10^{-5}$ for a SNR of $10 \mathrm{~dB}$, the required protection ratio is about $-18 \mathrm{~dB}$ if there is only one interferer. In presence of five interferer this value is $-20 \mathrm{~dB}$.

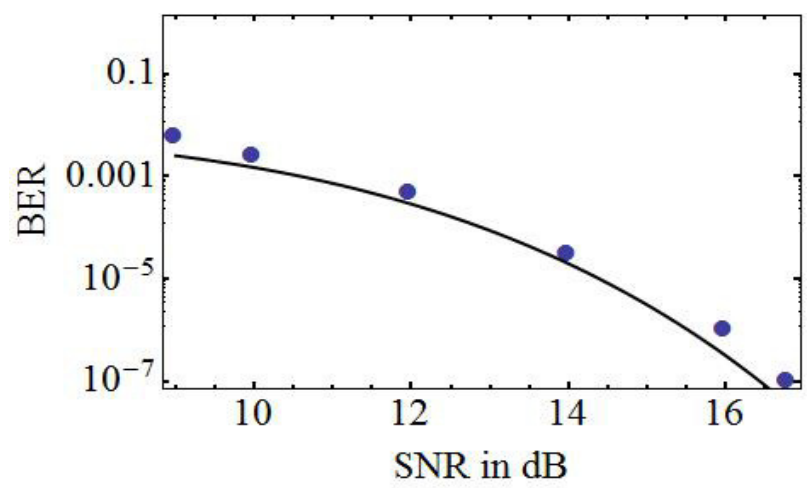

Fig.2:- Plot of BER versus SNR in $\mathrm{dB}$ for a crosstalk level of $-15 \mathrm{~dB}$ in presence of single interfering channels, where solid line represents present work and dot markers indicate result as per Fig. 4 of [25]. 


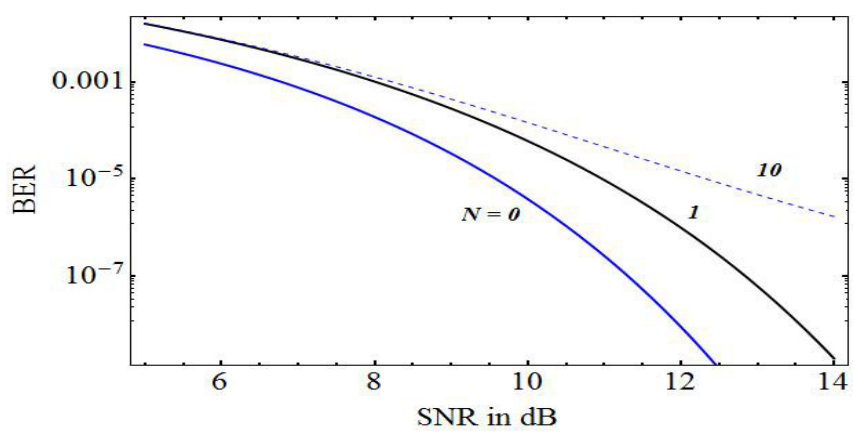

Fig.3:- Plot of BER versus SNR for a fixed crosstalk level of $-20 \mathrm{~dB}$ in presence of $N=0,1 \& 10$ interfering channels, keeping $t_{b}$ fixed at 1 second.

Spectral efficiency versus crosstalk level in presence of a fixed number of interferer $(N=1$, and 10) is shown in Fig. 5. The result shows that at a particular SNR value, presence of interfering channels with greater power deteriorates the spectral efficiency.

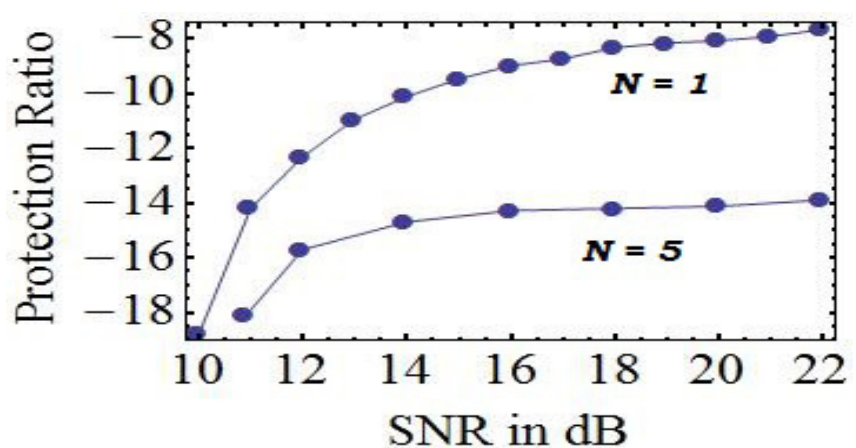

Fig.4:- Protection ratio required to obtain BER of $10^{-5}$ versus SNR for QPSK transmission in the presence of one and five interfering channels $(N)$ respectively.

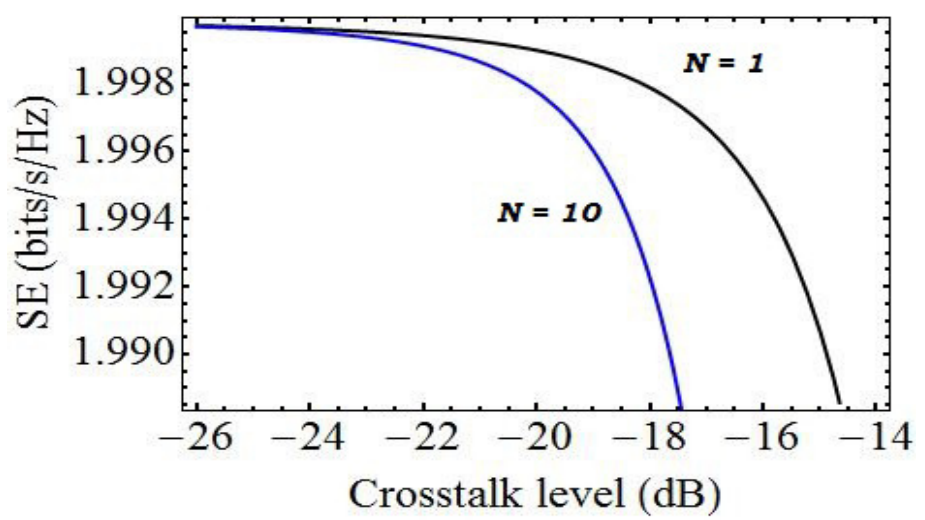

Fig.5:-.Spectral Efficiency versus crosstalk level for different interfering channels $(N=1$ and 10$)$ with SNR fixed at $10 \mathrm{~dB}$. 


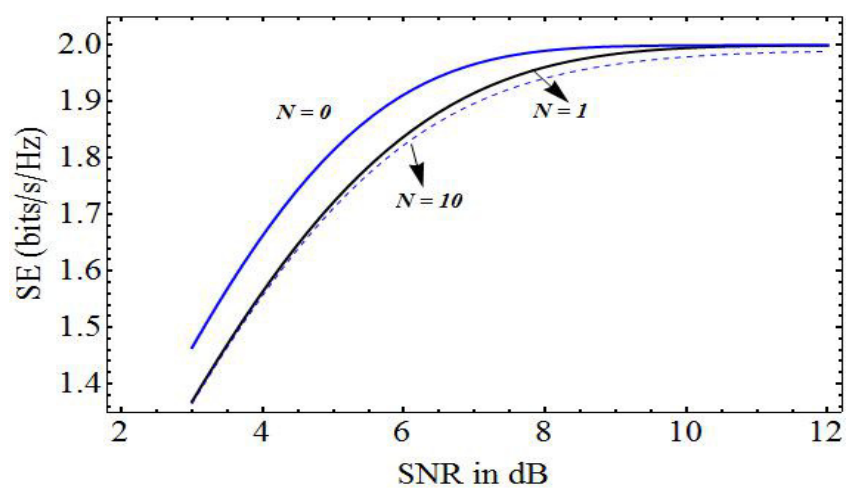

Fig.6:-.Spectral Efficiency versus SNR for different interfering channels $(N=0,1$ and 10$)$ with crosstalk level fixed at $-17.5 \mathrm{~dB}$.

Spectral efficiency (SE) versus SNR in presence of a fixed number of interferer $(N=0,1$ and 10) is shown in Fig. 6. The result shows that as the number of interfering channel increases, the efficiency decreases. It is also seen that SE is a non decreasing function of the SNR. Impacts of crosstalk sources are particularly prominent at the lower to mid SNR region. It is also clear that all modulations have a spectral efficiency at most $\log _{2}(M)$ bits $/ \mathrm{s} / \mathrm{Hz}$ (2 for QPSK as $M=4$ ) [27]. QPSK with Gray mapping is exactly twice spectrally efficient, compared to BPSK. In this context a parameter called SNR-Loss (i.e. additional SNR requirement to maintain same SE in presence of different crosstalk sources) is introduced which helps to understand the power budgeting of the network. To further elaborate this point, Fig. 7 is plotted where the SNR required to ensure spectral efficiency of 2 (BER of 10$\left.{ }^{9}\right)$ is shown in presence of a fixed number of interferer $(N=0,1,3,5$ and 10). The result shows that as the number of interfering channel increases, the SNR-Loss increases. For example, in presence of three interfering channel the required SNR is $13.85 \mathrm{~dB}$ whereas $14.47 \mathrm{~dB}$ is required for ten interferer (SNR-Loss of $1.92 \mathrm{~dB}$ ).

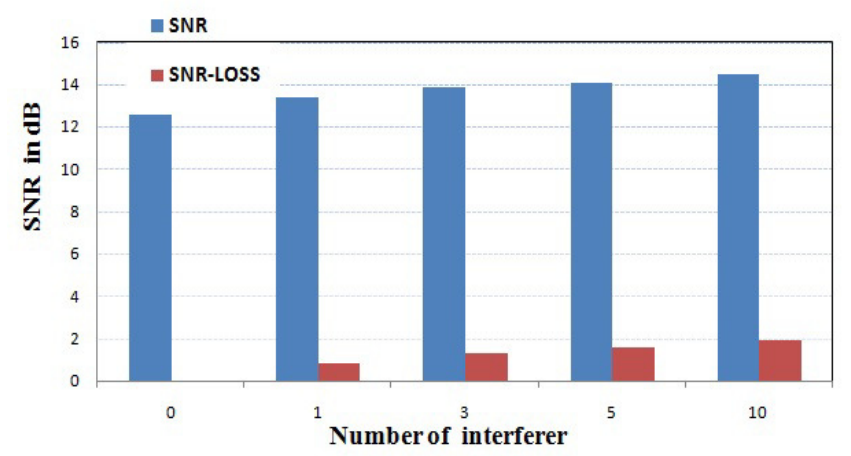

Fig.7:-.SNR as a function of interfering channels to ensure spectral efficiency of 2 (BER of $10^{-9}$ ) for different interfering channels with crosstalk level fixed at $-20 \mathrm{~dB}$.

Through Fig. 8, the variation of another performance criterion of interest, interference penalty is studied as a function of SNR. It shows the interfrence penalty plotted against SNR, in presence of various crosstalk sources $(N=1 \& 10)$ for a fixed crosstalk level of $-22 \mathrm{~dB}$. On 
the same graph variation of BER with respect to SNR is also shown. It is observed that interference penalty and BER follow opposite trend. As SNR increases penalty increases, whereas BER decreases. For example, Fig. 5 shows that for BER values of $\mathrm{P}_{\mathrm{e}}=9.01 \times 10^{-9}$, SNR values for with and without crosstalk are $14.33 \mathrm{~dB}$ and $12 \mathrm{~dB}$ respectively leading to interfrerence penalty of $0.77 \mathrm{~dB}$ for the case of ten dominant interferer $(N=10)$. With the help of Fig. 7 and Fig. 8, power budgeting of the network under component crosstalk, can be easily understood and altered to maintain same QoS (quality of service) with and without crosstalk sources.

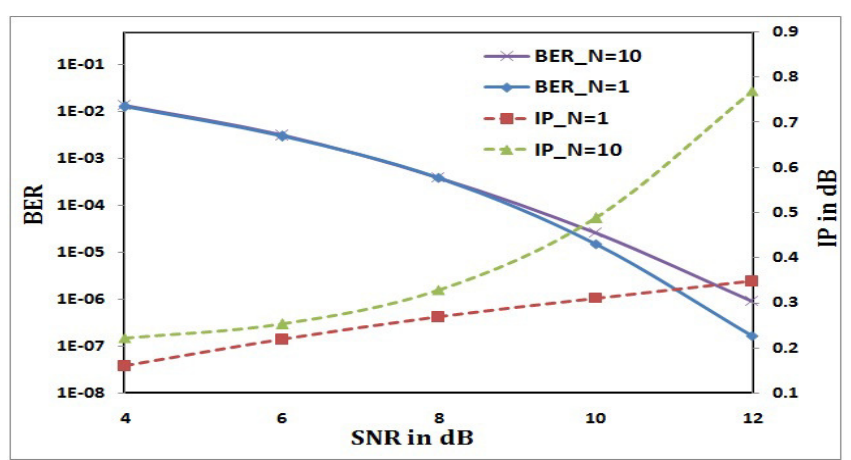

Fig 8:-BER and power penalty against SNR in presence of various interfering channels $(N)$, when $\varepsilon_{i}$ is fixed at $-22 \mathrm{~dB}$.

\section{CONCLUSION}

An analytical method is presented to investigate the impact of component crosstalk on the SE of generalized QPSK modulation format by computing its BER over AWGN channel. The analysis exploits characteristic function method and examines the bandwidth and power expenses of a WDM system while subjected to crosstalk sources. The precise results from previous literatures are used to assess the accuracies of the proposed model. It is observed from the result that crosstalk level and number of active interferers perform a vital role to determine the spectral efficiency of the system. It is also seen that SE is a non decreasing function of SNR and impact of crosstalk sources are particularly prominent at the low and mid SNR region. A parameter called SNR-Loss (i.e. additional SNR requirement to maintain same SE in presence of different crosstalk sources) is introduced and studied, which helps to understand the power budgeting of the network. The value of protection ratio (i.e. the minimum crosstalk level required to obtain a fixed BER), at any specific $S N R$ for any arbitrary crosstalk sources is also suggested. The assessment of this ratio is particularly important when the interference is caused by few crosstalk sources. The study of interference penalty reveals that BER and induced penalty follow opposite trend with respect to SNR. For 
a fixed crosstalk level, the interference penalty increases as the SNR increases while BER decreases.

\section{REFERENCES}

[1] Q. Zhang et al., "Accurate BER Estimation Scheme Based on K-Means Clustering Assisted Gaussian Approach for Arbitrary Modulation Format," in Journal of Lightwave Technology, vol. 38, no. 8, pp. 2152 2157, 15 April 15, 2020, doi: 10.1109/JLT.2019.2956804.

[2] F. Zhang et al., "Experimental Comparison of Different BER Estimation Methods for Coherent Optical QPSK Transmission Systems," in IEEE Photonics Technology Letters, vol. 23, no. 18, pp. 1343-1345, Sept.15, 2011, doi: 10.1109/LPT.2011.2160718.

[3] B. Krongold, T. Pfau, N. Kaneda and S. C. J. Lee, "Comparison Between PS-QPSK and PDM-QPSK With Equal Rate and Bandwidth," in IEEE Photonics Technology Letters, vol. 24, no. 3, pp. 203-205, Feb.1, 2012, doi: 10.1109/LPT.2011.2174978.

[4] J. Cheng, N. C. Beaulieu and X. Zhang, "Precise BER analysis of dual-channel reception of QPSK in Nakagami fading and cochannel interference," in IEEE Communications Letters, vol. 9, no. 4, pp. 316-318, April 2005, doi: 10.1109/LCOMM.2005.1413619.

[5] S. Sarkar and N. R. Das, "Study of Component Crosstalk and Obtaining Optimum Detection Threshold for Minimum Bit-Error-Rate in a WDM Receiver," J. Lightwave Tech., vol. 27, pp. 4366-4373, 2009.

[6] K.-P. Ho, Phase-Modulated Optical Communication Systems. New York: Springer-Verlag, 2005.

[7] E. L. Goldstein, L. Eskildsen and A. F. Elrefaie, "Performance implications of component crosstalk in transparent lightwave networks," in IEEE Photonics Technology Letters, vol. 6, no. 5, pp. 657-660, May 1994, doi: 10.1109/68.285571.

[8] P. J. Winzer, "High-Spectral-Efficiency Optical Modulation Formats," J. Lightwave Technol., Vol. 30, No. 24, pp. 3824-3835, 2012.

[9] D. S. Millar, T. Koike-Akino, S. Ö. Arık, K. Kojima, K. Parsons, T. Yoshida, and T. Sugihara, "Highdimensional modulation for coherent optical communications systems," Opt. Express, Vol. 22, No 7, pp. 8798-8812, 2014.

[10]Dalakas, V. and Kamalakis, "Comparative study of modulation and coding schemes for coherent indoor optical wireless systems,” Opt Quant Electron, vol. 48: 374, 2016.

[11] Pinakpani Mukherjee, S. Sarkar and N. R. Das, "An Approach for Realistic Estimation of BER due to Signal-Component Crosstalk in a WDM Receiver," Optik 146(2017)1-7.

[12] K. P. Ho, "Analysis of homodyne crosstalk in optical networks using Gram-Charlier Series", Journal of Lightwave Technology, vol.17, No.2, pp.149-154, 1999.

[13] T. Kamalakis and T. Sphicopoulos, "Asymptotic behavior of in-band crosstalk noise in WDM networks," IEEE Photon. Technol. Lett., vol. 15, no. 3, pp.476 -478, 2003.

[14]X. Fan, P. Bai, X. Liang, J. Zhang and B. Liu, "Detection Algorithm of BPSK Signal of ParameterAdjusted Bistable Stochastic Resonance Model Based on Scale Change," in IEEE Access, vol. 8, pp. 97643-97657, 2020, doi: 10.1109/ACCESS.2020.2997705. 
[15] A. M. Rabiei and N. Beaulieu, "An analytical expression for the BER of an individually optimal single cochannel interferer BPSK receiver", IEEE Trans. Commun., vol. 55, no. 1, pp. 60-63, 2007.

[16] A. M. Rabiei and N. C. Beaulieu, "A simple, intuitive expression for the BER of a jointly optimal single cochannel interferer BPSK receiver", IEEE Commun. Lett., vol. 9, no. 3, pp. 201-203, 2005.

[17] S. M. Saberali, H. Amindavar, and A. R. Moghaddamjoo, "BER calculation and investigation of optimal single user detector for a BPSK signal contaminated by cochannel interferer," IEEE Commun.Lett., vol. 12, no. 10, pp. 705-707, 2008.

[18]K. Chung, "An analytical expression for the BER of optimal single user detection of a BPSK signal contaminated by multiple CCIs," EURASIP J. Wirel. Commun. Netw., vol. 2012, no. 190, 2012.

[19]Darshi, S. and Bhattacharjee, "BER Analysis of Asynchronised Wireless Network in Presence of NonIdentically Distributed Interferers", Wireless PersCommun, vol. 82, pp 2583-2600, 2015.

[20] A. Giorgetti and M. Chiani, "Influence of fading on the Gaussian approximation for BPSK and QPSK with asynchronous cochannel interference," in IEEE Transactions on Wireless Communications, vol. 4, no. 2, pp. 384-389, March 2005, doi: 10.1109/TWC.2004.843036.

[21] M. Chiani and A. Giorgetti, "Statistical analysis of asynchronous QPSK cochannel interference," Global Telecommunications Conference, 2002. GLOBECOM '02. IEEE, 2002, pp. 1855-1859 vol.2, doi: 10.1109/GLOCOM.2002.1188520.

[22] M. Chiani, "Performance of BPSK and GMSK with multiple cochannel interferers," Proceedings of PIMRC '96 - 7th International Symposium on Personal, Indoor, and Mobile Communications, 1996, pp. 833-837 vol.3, doi: 10.1109/PIMRC.1996.568398.

[23] K. A. Hamdi and L. Pap, "Exact BER Analysis of Binary and Quaternary PSK With Generalized Selection Diversity in Cochannel Interference," in IEEE Transactions on Vehicular Technology, vol. 56, no. 4, pp. 1849-1856, July 2007, doi: 10.1109/TVT.2007.897238.

[24] N. C. Beaulieu, "The evaluation of error probabilities for intersymbol and cochannel interference," in IEEE Transactions on Communications, vol. 39, no. 12, pp. 1740-1749, Dec. 1991, doi: 10.1109/26.120161.

[25] N. C. Beaulieu and A. A. Abu-Dayya, "Bandwidth efficient QPSK in cochannel interference and fading," in IEEE Transactions on Communications, vol. 43, no. 9, pp. 2464-2474, Sept. 1995, doi: 10.1109/26.412721.

[26] K. P. Ho, "Effects of Homodyne Crosstalk on Dual-Polarization QPSK Signals," in Journal of Lightwave Technology, vol. 29, no. 1, pp.124-131, Jan.1, 2011, doi: 10.1109/JLT.2010.2095830.

[27]Taher, K. A. and Majumder, S. P., "Analytical Evaluation of the Effect of Cross-Polarization-induced Crosstalk on the BER Performance of a PDM-QPSK Coherent Homodyne Optical Transmission System”, $<\mathrm{i}>$ Journal of Optical Communications</i>, vol. 38, no. 2, pp. 213-222, 2017. doi:10.1515/joc-2016-0072.

[28] G. Kramer, A. Ashikhmin, A. J. van Wijngaarden and Xing Wei, "Spectral efficiency of coded phase-shift keying for fiber-optic communication," in Journal of Lightwave Technology, vol. 21, no. 10, pp. 24382445, Oct. 2003. doi: 10.1109/JLT.2003.817704.

[29] S. Verdu, "Spectral efficiency in the wideband regime," in IEEE Transactions on Information Theory, vol. 48, no. 6, pp. 1319-1343, June 2002. doi: 10.1109/TIT.2002.1003824.

[30] A. Baricz, "Powers of modified Bessel functions of the first kind", Applied Mathematics Letter, vol. 23, no. 6, pp. $722-724,2010$.

[31] A. Abramowitz and I. Stegun, Handbook of Mathematical functions, $9^{\text {th }}$ Ed. New York: Dover, 1972. 
[32] A. Papoulis, Probability, Random Variable, and Stochastic Processes, New York: McGraw-Hill, 1984.

[33] J. G. Proakis, Digital Communications, New York: McGraw-Hill, 2000.

\section{Statements \& Declarations:}

\section{Funding}

The authors declare that no funds, grants, or other support were received during the preparation of this manuscript.

\section{Competing Interests}

The authors have no relevant financial or non-financial interests to disclose.

\section{Author Contributions}

Pinakpani Mukherjee (PPM) did the analysis and plotted the results. Santu Sarkar (SS) provided the idea behind the choice of the topic. Nikhil Ranjan Das (NRD) checked the analysis and supervised the whole work. All authors read and approved the final manuscript.

\section{Data Availability}

Any applicable data are available in the main manuscript. No other associated data applicable. 